\title{
CRACK-LIKE IMPERFECTIONS IN A SPHERICAL SHELL $\dagger$
}

\author{
by G. C. SIH and P. S. DOBREFF
}

(Received 26 April, 1969)

Introduction. In structures having high strength-to-weight ratios such as those used in aerospace applications, the presence of mechanical imperfections can reduce the capability of the structure to perform as intended. Thus, it becomes essential to account for the localized intensification of the stresses around through or surface cracks, which might trigger fracture under applied loads. This type of study is currently receiving great research emphasis.

Although a considerable amount of effort has been expended on the stress analysis of cracks in initially flat plates subjected to either extensional and/or bending loads (a review of which can be found in [1]), theoretical treatment of cracks in initially curved plates or shells has not received adequate attention in the past. The presence of curvature in a shell generates deviation from behaviour of flat plates, in that stretching loads will induce both extensional and bending stresses, while bending loads will also lead to both types of stresses. One of the simplest shell geometries is that of a spherical shell, where the curvature radius $R$ is everywhere constant. The first investigation on the stresses in a spherical shell containing a crack was made by Ang et al. [2], who associated the problem with that of an initially flat plate resting upon an elastic foundation. The equivalence of the two problems was made by identifying the foundation modulus with $E h / R^{2}$ where $E$ is the Young's modulus and $h$ the shell thickness. However, the general character of the crack-tip stress field in a shell was not well understood until Sih and Setzer [3] pointed out that the functional relationships of the local extensionbending stresses are identical with those obtained by superimposing the individual extensional and bending stresses of an initially flat plate. The extensional and bending effects are interlaced only through the intensity of the local stress field. In another paper, Folias [4] gave a separate treatment to the spherical shell problem by utilizing singular integral equations with Cauchy type kernels, as devised by Knowles and Wang [5], for solving crack problems involving flat plates. The formulation in [4] $\$$ relies upon certain approximations in the kernels for small $\lambda a=\left[12\left(1-v^{2}\right)\right]^{\frac{1}{1}} a /(R h)^{\frac{1}{2}}$ and assumptions on the behaviour of the density functions based on the known flat plate solution. As a result, the obtained solution is valid only for an extremely narrow range of $\lambda a$. This will be illustrated in the present work.

In order to extend the results of [4] to cover a significant range of $\lambda a$, a new method for handling shell problems with cracks is presented. With the aid of Fourier transforms, an integral representation for the solution of the governing differential equations of a spherical shell is obtained. Application of the boundary conditions reduces the problem to two coupled Fredholm integral equations in two unknown functions, which are solved numerically with a high degree of accuracy. One of the advantages of the method is that the singular portion of the stress solution can be readily extracted from the density functions and determined in closed

† This study was supported by the National Aeronautics and Space Administration under Grant NGR-39007-025 with Lehigh University.

$¥$ It should be noted that the parameter $\lambda$ used in this paper is equal to $\lambda / a$ in [4].

E 
elementary form. Numerical results for the stresses and displacements in a cracked spherical shell are also displayed graphically.

Shallow shell equations. The linear, first-order (thin) shallow shell theory will be employed with the assumptions that the crack is not too long compared with the radius of curvature and that the shallowness of the shell allows terms of order $(H / L)^{2}$ to be neglected in comparison with unity. The parameters $H$ and $L$ are explained in Fig. 1 . The points in the shell will be

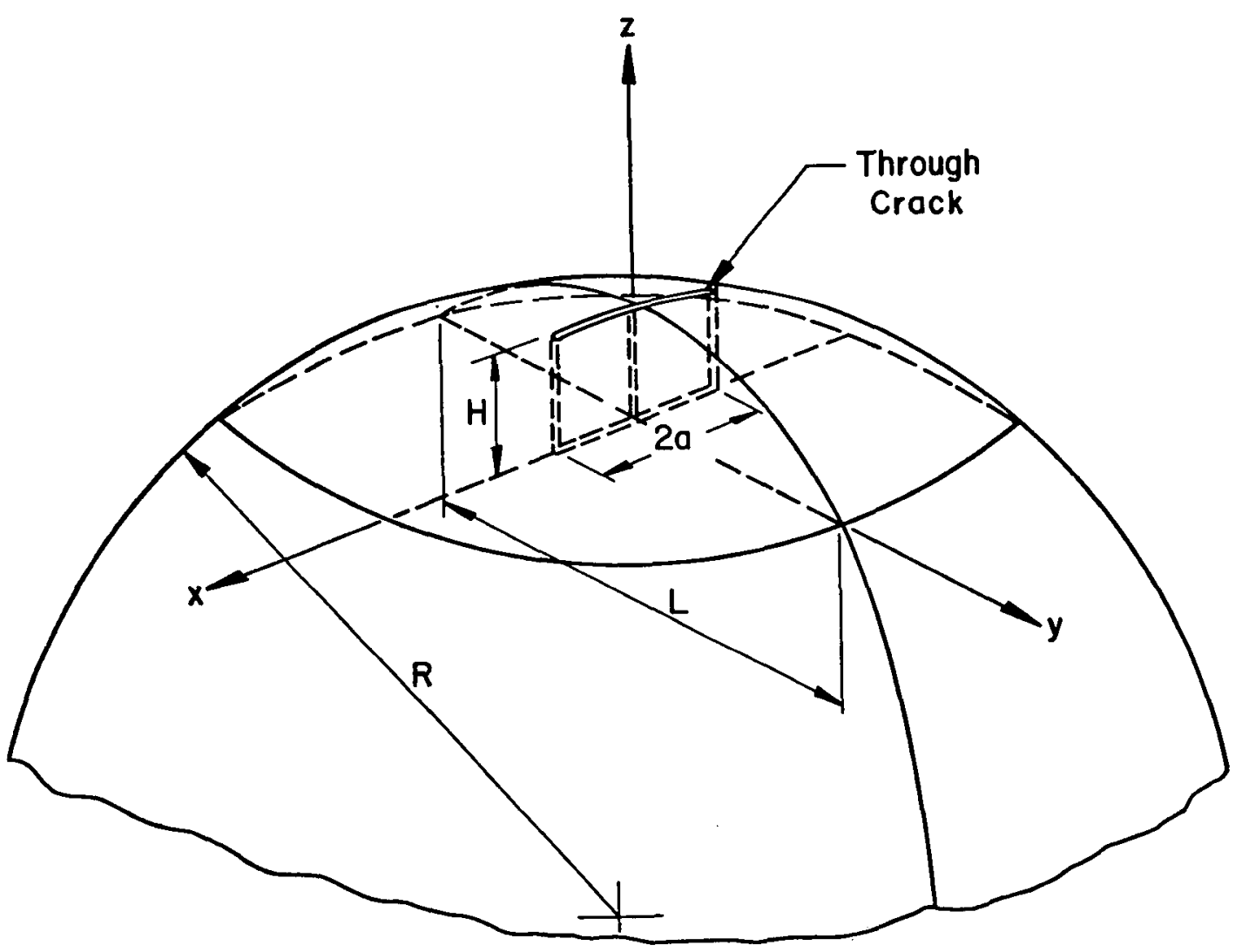

Fig. 1. Geometry of Shallow Spherical Shell with a Crack at the Apex.

identified by the coordinates of their projections in the $x y$-plane so that a crack lying at the apex of the shell may be taken as the segment $y=0,|x| \leqq a$.

By the shallow shell theory of Reissner [6], the coupled differential equations are

$$
\nabla^{4} w+\frac{1}{R D} \nabla^{2} \Phi=p(x, y) / D, \quad \nabla^{4} \Phi-\frac{E h}{R} \nabla^{2} w=0,
$$


where $w(x, y)$ is the normal displacement and $\Phi(x, y)$ is the stress function, which is identical in character to the Airy stress function used in generalized plane stress problems. In (1), $\nabla^{2}$ is the Laplacian operator $\partial^{2} / \partial x^{2}+\partial^{2} / \partial y^{2}, D$ is the flexural rigidity of the shell defined as $E h^{3} / 12\left(1-v^{2}\right)$, and $p(x, y)$ is the normal loading applied to the concave side of the shell. The Poisson's ratio is denoted by $v$. As usual, the membrane stress resultants are derived from $\Phi(x, y)$ as

$$
N_{x}=\frac{\partial^{2} \Phi}{\partial y^{2}}, \quad N_{y}=\frac{\partial^{2} \Phi}{\partial x^{2}}, \quad N_{x y}=-\frac{\partial^{2} \Phi}{\partial x \partial y} .
$$

The constitutive relations expressing the moments and transverse shear resultants in terms of the normal displacement are

$$
M_{x}=-D\left(\frac{\partial^{2} w}{\partial x^{2}}+v \frac{\partial^{2} w}{\partial y^{2}}\right), \quad M_{y}=-D\left(\frac{\partial^{2} w}{\partial y^{2}}+v \frac{\partial^{2} w}{\partial x^{2}}\right), \quad M_{x y}=-D(1-v) \frac{\partial^{2} w}{\partial x \partial y}
$$

and

$$
Q_{x}=-D \frac{\partial}{\partial x}\left(\nabla^{2} w\right), \quad Q_{y}=-D \frac{\partial}{\partial y}\left(\nabla^{2} w\right)
$$

From the ordinary strain-stress relations, the in-plane displacements $u(x, y)$ and $v(x, y)$ can be obtained in the usual manner.

Since the first-order shell theory permits only four conditions at an edge, say $y=0$, the quantities $Q_{y}$ and $M_{x y}$ cannot be specified individually, but they must be combined in the Kirchhoff sense, i.e.,

$$
\left.\begin{array}{l}
V_{x}=Q_{x}+\frac{\partial M_{x y}}{\partial y}=-D\left[\frac{\partial^{3} w}{\partial x^{3}}+(2-v) \frac{\partial^{3} w}{\partial x \partial y^{2}}\right] \\
V_{y}=Q_{y}+\frac{\partial M_{x y}}{\partial x}=-D\left[\frac{\partial^{3} w}{\partial y^{3}}+(2-v) \frac{\partial^{3} w}{\partial x^{2} \partial y}\right]
\end{array}\right\}
$$

Hence, the admissible boundary conditions at the edge $y=0$ consist of selecting one member from each of the following pairs:

$$
\left(M_{y}, \frac{\partial w}{\partial y}\right),\left(V_{y}, w\right), \quad\left(N_{y}, v\right), \quad\left(N_{x y}, u\right) .
$$

Further, if the problem is symmetric about the $x z$-plane, then the additional conditions

$$
\frac{\partial w}{\partial y}=v=V_{y}=N_{x y}=0 \text { for } y=0
$$

can be used to simplify the mathematical analysis.

Preliminary remarks and calculations. In treating this problem, the solution may be divided into two parts, namely the " undisturbed " solution for the shell without a crack which can be computed from (1) for a particular function $p(x, y)$ but leaves residual moments and 
membrane stresses along the prospective crack site, and a "perturbation " solution, which nullifies these residuals and decays rapidly as distance from the crack is increased. The rate of decay will depend on the magnitude of the parameter $\lambda a$ and is taken to be sufficiently rapid such that the perturbation solution is effectively zero, except in a small region surrounding the crack within which the shell is shallow. This will be loosely referred to as the regularity condition, which requires $w(x, y)$ and $\Phi(x, y)$ with their first derivatives to be bounded as $\left(x^{2}+y^{2}\right)^{\frac{1}{2}} \rightarrow \infty$. The solution for the uncracked shell can usually be found without difficulty. Therefore, the major task of the shell problem lies in determining the perturbed stress field which corresponds to the homogeneous solution of (1), i.e., with $p(x, y)=0$.

For the purpose of demonstrating the essential results of a cracked shell, it suffices to consider the perturbation solution for the case when the crack is opened out symmetrically $\dagger$ with respect to the $x z$-plane. Owing to the conditions stated in (7) and assumptions made on the decay of the perturbed stress solution, an equivalent problem involving a semi-infinite shell occupying the space $y \geqq 0$ may be set up with mixed boundary conditions along the edge $y=0$. More specifically, the conditions on the crack at $y=0$ are

$$
\left.\begin{array}{rl}
M_{y}(x, 0) & =M(x), \quad V_{y}(x, 0)=0, \\
N_{y}(x, 0) & =N(x), \quad N_{x y}(x, 0)=0 .
\end{array}\right\}
$$

For $|x|<a$ and along the rest of the line $y=0$, it is necessary to have

$$
\lim _{y \rightarrow 0}\left(\frac{\partial w}{\partial y}\right)=v(x, 0)=V_{y}(x, 0)=N_{x y}(x, 0)=0 \text { for }|x|>a
$$

Note, from the above expressions, that $V_{y}$ and $N_{x y}$ vanish for all values of $x$ at $y=0$.

The coupled shallow shell equations can be conveniently solved by application of the Fourier cosine and sine transforms

$$
f_{1}^{c}(s, y)=\int_{0}^{\infty} f_{1}(x, y) \cos (s x) d x, \quad f_{2}^{\mathrm{s}}(s, y)=\int_{0}^{\infty} f_{2}(x, y) \sin (s x) d x,
$$

in which $s$ is the real-valued transform parameter. According to the appropriate inversion theorem [7], (10) implies that

$$
f_{1}(x, y)=\frac{2}{\pi} \int_{0}^{\infty} f_{1}^{c}(s, y) \cos (s x) d s, \quad f_{2}(x, y)=\frac{2}{\pi} \int_{0}^{\infty} f_{2}^{s}(s, y) \sin (s x) d s .
$$

Assuming that the displacement function $w(x, y)$ and the stress function $\Phi(x, y)$ satisfy the regularity condition as mentioned earlier and applying (10), we find that the solution to (1) in the transformed domain is given by

$$
\left.\begin{array}{l}
w^{c}(s, y)=F_{1}(s) \exp (-s \alpha y)+F_{2}(s) \exp (-s \beta y)+F_{3}(s) \exp (-s y) \quad(y \geqq 0), \\
\Phi^{c}(s, y)=i \lambda^{2} R D\left[F_{1}(s) \exp (-s \alpha y)-F_{2}(s) \exp (-s \beta y)+F_{4}(s) \exp (-s y)\right] \quad(y \geqq 0),
\end{array}\right\}
$$

The formulation of the anti-symmetric problem follows along the same line of reasoning and will not be dealt with here. 
in which the parameters $\alpha, \beta$ and $\lambda$ stand for

$$
\alpha(s)=\left[1-i(\lambda / s)^{2}\right]^{\frac{1}{2}}, \quad \beta(s)=\left[1+i(\lambda / s)^{2}\right]^{\frac{1}{2}}, \quad \lambda=\left[12\left(1-v^{2}\right)\right]^{\frac{1}{}}(R h)^{-\frac{1}{t}} .
$$

Similarly, appropriate sine and cosine transforms may be applied to the quantities in equations (2) to (5). In terms of the functions $F_{j}(s)(j=1,2, \ldots, 4)$, the equations (2) for $y \geqq 0$ are

$$
\left.\begin{array}{rl}
N_{x}^{c} & =i(\lambda s)^{2} R D\left[\alpha^{2} F_{1}(s) \exp (-s \alpha y)-\beta^{2} F_{2}(s) \exp (-s \beta y)+F_{4}(s) \exp (-s y)\right], \\
N_{y}^{c} & =-i(\lambda s)^{2} R D\left[F_{1}(s) \exp (-s \alpha y)-F_{2}(s) \exp (-s \beta y)+F_{4}(s) \exp (-s y)\right], \\
N_{x y}^{s} & =-i(\lambda s)^{2} R D\left[\alpha F_{1}(s) \exp (-s \alpha y)-\beta F_{2}(s) \exp (-s \beta y)+F_{4}(s) \exp (-s y)\right],
\end{array}\right\}
$$

and the equations (3) become

$$
\left.\begin{array}{rl}
M_{x}^{c}= & s^{2} D\left[\left(1-v \alpha^{2}\right) F_{1}(s) \exp (-s \alpha y)+\left(1-v \beta^{2}\right) F_{2}(s) \exp (-s \beta y)\right. \\
& \left.+(1-v) F_{3}(s) \exp (-s y)\right], \\
M_{y}^{c}= & s^{2} D\left[\left(v-\alpha^{2}\right) F_{1}(s) \exp (-s \alpha y)+\left(v-\beta^{2}\right) F_{2}(s) \exp (-s \beta y)\right. \\
& \left.-(1-v) F_{3}(s) \exp (-s y)\right], \\
M_{x y}^{s}= & -(1-v) s^{2} D\left[\alpha F_{1}(s) \exp (-s \alpha y)+\beta F_{2}(s) \exp (-s \beta y)+F_{3}(s) \exp (-s y)\right],
\end{array}\right\}
$$

which are valid only for $y \geqq 0$. The Fourier transforms of the Kirchhoff shear resultants take the forms

$$
\left.\begin{array}{rl}
V_{x}^{s}= & s^{3} D\left\{\left[(2-v) \alpha^{2}-1\right] F_{1}(s) \exp (-s \alpha y)+\left[(2-v) \beta^{2}-1\right] F_{2}(s) \exp (-s \beta y)\right. \\
& \left.+(1-v) F_{3}(s) \exp (-s y)\right\}, \\
V_{y}^{c}= & s^{3} D\left\{\alpha\left[\alpha^{2}-(2-v)\right] F_{1}(s) \exp (-s \alpha y)+\beta\left[\beta^{2}-(2-v)\right] F_{2}(s) \exp (-s \beta y)\right. \\
& \left.-(1-v) F_{3}(s) \exp (-s y)\right\},
\end{array}\right\}
$$

for $y \geqq 0$ and the in-plane displacements $u$ and $v$ are transformed into

$$
\begin{aligned}
E h u^{s}= & i \lambda^{2} s R D\left[\left(\alpha^{2}+v\right) F_{1}(s) \exp (-s \alpha y)-\left(\beta^{2}+v\right) F_{2}(s) \exp (-s \beta y)\right. \\
& \left.+(1+v) F_{4}(s) \exp (-s y)\right] \quad(y \geqq 0), \\
E h v^{c}= & i \lambda^{2} s R D\left\{\alpha\left[\alpha^{2}-(2-v)\right] F_{1}(s) \exp (-s \alpha y)+\left[\beta^{2}-(2-v)\right] F_{2}(s) \exp (-s \beta y)\right. \\
& \left.-(1+v) F_{4}(s) \exp (-s y)\right\} \quad(y \geqq 0) .
\end{aligned}
$$

The above expressions $N_{x}^{c}, N_{y}^{c}$, etc. can be easily inverted back to the physical domain by means of (11).

At this point, the number of unknown functions $F_{j}(s)(j=1,2, \ldots, 4)$ may be reduced by appealing to the symmetry conditions on $V_{y}$ and $N_{x y}$ in (8) and (9). Since

$$
V_{y}^{c}(s, 0)=N_{x y}^{s}(s, 0)=0
$$

the third equation in (14) and the second equation in (16) may be used to eliminate $F_{j}(s)$ E2 
$(j=3,4)$ as follows:

$$
\begin{aligned}
(1-v) F_{3}(s) & =-\alpha\left(\beta^{2}-v\right) F_{1}(s)-\beta\left(\alpha^{2}-v\right) F_{2}(s), \\
F_{4}(s) & =-\alpha F_{1}(s)+\beta F_{2}(s) .
\end{aligned}
$$

Hence, equations (14) to (17) may be expressed in terms of only two unknowns $F_{j}(s)(j=1,2)$. From the remaining boundary conditions specified in (8), (9) and the first equation (11), $F_{j}(s)(j=1,2)$ may be shown to be governed by the coupled dual integral equations

$$
\left.\begin{array}{ll}
\int_{0}^{\infty}(\alpha / s) F_{1}(s) \cos (s x) d s=0 & (x>a), \\
\int_{0}^{\infty}(\beta / s) F_{2}(s) \cos (s x) d s=0 & (x>a),
\end{array}\right\}
$$

and

$$
\begin{aligned}
& \int_{0}^{\infty}\left\{\left[\alpha\left(\alpha-\beta^{2}\right)-v(1-\alpha)\right] F_{1}(s)+\left[\beta\left(\beta-\alpha^{2}\right)-v(1-\beta)\right] F_{2}(s)\right\} s^{2} \cos (s x) d s \\
& =-\frac{1}{2} \pi m(x) \quad(x<a), \\
& \int_{0}^{\infty}\left[(1-\alpha) F_{1}(s)-(1-\beta) F_{2}(s)\right] s^{2} \cos (s x) d s=i \frac{1}{2} \pi n(x) \quad(x<a),
\end{aligned}
$$

in which

$$
m(x)=\frac{M(x)}{D}, \quad n(x)=\frac{N(x)}{\lambda^{2} R D}
$$

It should be noted that the present problem is also symmetric about the $y z$-plane.

While the analysis leading up to the foregoing integral representations is somewhat straightforward, it is offered here merely for the sake of completeness. The reduction of equations (19) and (20) to a system of standard integral equations which are suitable for numerical evaluation will now be carried out.

Reduction of coupled dual integral equations. One approach to the mixed boundary problem described herein is to reduce (19) and (20) to two coupled integral equations, which can be evaluated along the crack $-a<x<a$. To this end, the functions $u_{j}(x)(j=1,2)$ are introduced:

$$
u_{1}(x)=\frac{2}{\pi} \int_{0}^{\infty}(\alpha / s) F_{1}(s) \cos (s x) d s, \quad u_{2}(x)=\frac{2}{\pi} \int_{0}^{\infty}(\beta / s) F_{2}(s) \cos (s x) d s .
$$

As is apparent from $(19), u_{j}(x)(j=1,2)$ vanish for $x>a$ and hence the Fourier inversion theorem [7] yields

$$
(\alpha / s) F_{1}(s)=\int_{0}^{a} u_{1}(x) \cos (s x) d x, \quad(\beta / s) F_{2}(s)=\int_{0}^{a} u_{2}(x) \cos (s x) d x .
$$


In order to avoid certain difficulties associated with the singularities that arise in the solution for a line crack, it is essential to restrict the behaviour of the crack opening displacements or the functions $u_{j}(x)$ at the end-points $x= \pm a$. Guided by the form of $u_{j}(x)$ being proportional to $\left(a^{2}-x^{2}\right)^{\frac{1}{2}}$ for the flat plate solution [8] $\lambda=0$, we may take the desired representations to be

$$
u_{j}(x)=\left\{\begin{array}{cc}
\int_{x}^{a} \psi_{j}(t) \frac{t d t}{\sqrt{\left(t^{2}-x^{2}\right)}} & (x<a), \\
0 & (x>a),
\end{array}\right\}
$$

for $j=1,2$. Now, inserting (23) into (22) and making use of the identity

$$
\int_{0}^{t} \frac{\cos (s x)}{\sqrt{\left(t^{2}-x^{2}\right)}} d x=\frac{1}{2} \pi J_{0}(s t)
$$

where $J_{0}$ is the zero-order Bessel function of the first kind, we see that the functions $F_{j}(s)$ in (20) may thus be replaced by $\psi_{j}(t)$. The result of integrating (20) once in $x$ gives

$$
\left.\begin{array}{l}
\int_{0}^{\infty}\left[p_{1}(s) \int_{0}^{a} \psi_{1}(t) J_{0}(s t) t d t+\bar{p}_{1}(s) \int_{0}^{a} \psi_{2}(t) J_{0}(s t) t d t\right] \sin (s x) d s=-\int_{0}^{x} m(\eta) d \eta \\
\int_{0}^{\infty}\left[p_{2}(s) \int_{0}^{a} \psi_{1}(t) J_{0}(s t) t d t-\bar{p}_{2}(s) \int_{0}^{a} \psi_{2}(t) J_{0}(s t) t d t\right] \sin (s x) d s=i \int_{0}^{x} n(\eta) d \eta
\end{array}\right\}
$$

in which $\bar{p}_{j}(s)(j=1,2)$ are the complex conjugate of $p_{j}(s)$. In the sequel, the overbar will be used to denote the complex conjugate of a function.

The next objective is to reduce (24) to a system of coupled Fredholm equations of the second kind. For this purpose, introduce

$$
q_{j}(s)=p_{j}(s)-i \alpha_{j} \quad(j=1,2),
$$

with real

$$
\alpha_{1}=-(3+v) \lambda^{2} / 2 \text { and } \alpha_{2}=\lambda^{2} / 2
$$

such that $q_{j}(s)$ is of order $s^{-2}$ as $s \rightarrow \infty$. Now, by virtue of the discontinuous integral [9]

$$
\int_{0}^{a} J_{0}(s t) \sin (s x) d s=\left\{\begin{array}{cc}
0 & (0 \leqq x<t), \\
\left(x^{2}-t^{2}\right)^{-\frac{1}{2}} & (0<t<x)
\end{array}\right.
$$

(24) may be written as

$$
\begin{aligned}
\int_{0}^{x} i \alpha_{1}\left[\psi_{1}(t)-\psi_{2}(t)\right] \frac{t d t}{\sqrt{\left(x^{2}-t^{2}\right)}=-\int_{0}^{x} m(\eta) d \eta}-\int_{0}^{a} \psi_{1}(t) t d t \int_{0}^{\infty} q_{1}(s) J_{0}(s t) \sin (s x) d s \\
-\int_{0}^{a} \psi_{2}(t) t d t \int_{0}^{\infty} \bar{q}_{1}(s) J_{0}(s t) \sin (s x) d s
\end{aligned}
$$


and

$$
\begin{aligned}
\int_{0}^{x} i \alpha_{2}\left[\psi_{1}(t)+\psi_{2}(t)\right] \frac{t d t}{\sqrt{\left(x^{2}-t^{2}\right)}=i \int_{0}^{x} n(\eta) d \eta} & -\int_{0}^{a} \psi_{1}(t) t d t \int_{0}^{\infty} q_{2}(s) J_{0}(s t) \sin (s x) d s \\
& +\int_{0}^{a} \psi_{2}(t) t d t \int_{0}^{\infty} \bar{q}_{2}(s) J_{0}(s t) \sin (s x) d s
\end{aligned}
$$

These are recognized as Abel integral equations of the special type $\dagger$ :

$$
\int_{0}^{x} H(t) \frac{t d t}{\sqrt{\left(x^{2}-t^{2}\right)}}=h(x) \quad(0 \leqq x<a),
$$

and the function $H(t)$ can be inverted as

$$
H(t)=\frac{2}{\pi} \int_{0}^{t} \frac{d}{d x}[h(x)] \frac{d x}{\sqrt{\left(t^{2}-x^{2}\right)}} \quad(0 \geqq t>a) .
$$

Applying (27) to (25) and introducing the dimensionless quantities

$$
\xi=t / a, \quad \eta=\tau / a, \quad \Psi_{j}(\xi)=\sqrt{ }(\xi) \psi_{j}(a \zeta) \quad(j=1,2),
$$

we immediately obtain

$$
\left.\begin{array}{rl}
i \alpha_{1}\left[\Psi_{1}(\xi)-\Psi_{2}(\xi)\right] & =-\frac{2}{\pi} \sqrt{ }(\xi) \int_{0}^{\xi} \frac{m(x a) d x}{\sqrt{\left(\xi^{2}-x^{2}\right)}}-\int_{0}^{1}\left[P_{1}(\xi, \eta) \Psi_{1}(\eta)+\bar{P}_{1}(\xi, \eta) \Psi_{2}(\eta)\right] d \eta, \\
i \alpha_{2}\left[\Psi_{1}(\xi)+\Psi_{2}(\xi)\right] & =i \frac{2}{\pi} \sqrt{ }(\xi) \int_{0}^{\xi} \frac{n(x a) d x}{\sqrt{ }\left(\xi^{2}-x^{2}\right)}-\int_{0}^{1}\left[P_{2}(\xi, \eta) \Psi_{1}(\eta)-\bar{P}_{2}(\xi, \eta) \Psi_{2}(\eta)\right] d \eta,
\end{array}\right\}
$$

for $0 \leqq \xi \leqq 1$. Here, the symmetric kernels

$$
P_{j}(\xi, \eta)=\sqrt{ }(\xi \eta) \int_{0}^{\infty} s q_{j}(s / a) J_{0}(\xi s) J_{0}(\eta s) d s \quad(0 \leqq \xi \leqq 1,0 \leqq \eta \leqq 1),
$$

for $j=1,2$, are continuous on the square domain of definition $[0,1] \times[0,1]$ and are positive definite. By splitting the kernels in (29) as

$$
P_{1}(\xi, \eta)=\alpha_{1}\left[Q_{11}(\xi, \eta)+i Q_{12}(\xi, \eta)\right], \quad P_{2}(\xi, \eta)=\alpha_{2}\left[Q_{21}(\xi, \eta)+i Q_{22}(\xi, \eta)\right]
$$

and defining

$$
\phi_{1}(\xi)=i\left[\Psi_{1}(\xi)-\Psi_{2}(\xi)\right], \quad \phi_{2}(\xi)=\Psi_{1}(\xi)+\Psi_{2}(\xi)
$$

$\dagger$ With the change of variables $X=x^{2}$ and $T=t^{2},(26)$ and (27) can be readily converted to the regular pair of Abel integral equations [10]

$$
\int_{0}^{X} \frac{F(T) d T}{\sqrt{ }(X-T)}=f(X), \quad F(T)=\frac{1}{\pi} \int_{0}^{T} \frac{d}{d X}[f(X)] \frac{d X}{\sqrt{ }(T-X)}
$$


the following system of coupled Fredholm integral equations is found:

$$
\begin{array}{ll}
\phi_{1}(\xi)+\int_{0}^{1}\left[Q_{11}(\xi, \eta) \phi_{2}(\eta)+Q_{12}(\xi, \eta) \phi_{1}(\eta)\right] d \eta=-\frac{2 \sqrt{ }(\xi)}{\pi \alpha_{1}} \int_{0}^{\xi} \frac{m(x a) d x}{\sqrt{\left(\xi^{2}-x^{2}\right)}}, & (0 \leqq \xi<1) \\
\phi_{2}(\xi)-\int_{0}^{1}\left[Q_{21}(\xi, \eta) \phi_{1}(\eta)-Q_{22}(\xi, \eta) \phi_{2}(\eta)\right] d \eta=\frac{2 \sqrt{ }(\xi)}{\pi \alpha_{2}} \int_{0}^{\xi} \frac{n(x a) d x}{\sqrt{\left(\xi^{2}-x^{2}\right)}} &
\end{array}
$$

where $Q_{j k}(\xi, \eta)(j, k=1,2)$ is given by

$$
\left.\begin{array}{l}
Q_{j 1}(\xi, \eta)=\operatorname{Re}\left[\alpha_{j}^{-1} \sqrt{ }(\xi \eta) \int_{0}^{\infty} s q_{j}(s / a) J_{0}(\xi s) J_{0}(\eta s) d s\right], \\
Q_{j 2}(\xi, \eta)=\operatorname{Im}\left[\alpha_{j}^{-1} \sqrt{ }(\xi \eta) \int_{0}^{\infty} s q_{j}(s / a) J_{0}(\xi s) J_{0}(\eta s) d s\right],
\end{array}\right\}
$$

for $0 \leqq \xi \leqq 1,0 \leqq \eta \leqq 1$. Equations (31) and (32) render $\phi_{j}(\xi)(j=1,2)$ fully determinate once $m(x)$ and $n(x)$ are specified on the crack surface. The case of constant moment $m_{0}$ and stress resultant $n_{0}$ applied to the crack will be considered for numerical calculation later on.

The improper integral representations for $Q_{j k}(\xi, \eta)(j=1,2)$ in (32), however, are inconvenient for numerical purposes because of the infinite range of integration and the oscillatory character of the integrands concerned. Alternative representations, which improve the rate of convergence of the numerical solution, are readily deduced by expressing $q_{j}(s)$ in (32) in terms of $g_{j}(s)$, i.e.,

$$
g_{j}(s)=\frac{c_{j}}{s^{2}-i n_{j}^{2}}-q_{j}(s) \quad(j=1,2),
$$

so that $g_{j}(s)=O\left(s^{-6}\right)$ as $s \rightarrow \infty$. In (33), the constants $c_{j}$ and $n_{j}^{2}$ are

$$
c_{1}=\frac{1+3 v}{8} \lambda^{4}, \quad c_{2}=-\frac{3}{8} \lambda^{4}
$$

and

$$
n_{1}^{2}=\frac{1+5 v}{2(1+3 v)} \lambda^{2}, \quad n_{2}^{2}=\frac{5}{6} \lambda^{2}
$$

On putting (33) into (32) and using the identities [9]

$$
\int_{0}^{\infty} \frac{s}{s^{2}-i n^{2}} J_{0}(\xi s) J_{0}(\eta s) d s=-i \frac{1}{2} \pi J_{0}(\sqrt{i \xi n}) H_{0}^{(1)}(\sqrt{i \eta n}) \quad(0<\xi \leqq \eta),
$$

in which $J_{0}$ and $H_{0}^{(1)}$ can be expressed in terms of the Kelvin functions ber, bei, ker and kei as

$$
J_{0}(\sqrt{i x})=\operatorname{ber} x-i \text { bei } x, \quad-i \frac{1}{2} \pi H_{0}^{(1)}(\sqrt{i x})=\operatorname{ker} x-i \operatorname{kei} x,
$$


the kernels $Q_{j k}(\xi, \eta)$ become, for $0 \leqq \xi \leqq 1,0 \leqq \eta \leqq 1$,

$$
\begin{gathered}
Q_{j 1}(\xi, \eta)=\alpha_{j}^{-1} \sqrt{ }(\xi \eta)\left\{a^{2} c_{j}\left[\operatorname{ber}\left(a \xi n_{j}\right) \operatorname{ker}\left(a \eta n_{j}\right)-\operatorname{bei}\left(a \xi n_{j}\right) \operatorname{kei}\left(a \eta n_{j}\right)\right]\right. \\
\left.-\operatorname{Re}\left[\int_{0}^{\infty} s g_{j}(s / a) J_{0}(\xi s) J_{0}(\eta s) d s\right]\right\} \\
Q_{j 2}(\xi, \eta)=-\alpha_{j}^{-1} \sqrt{ }(\xi \eta)\left\{a^{2} c_{j}\left[\operatorname{ber}\left(a \xi n_{j}\right) \operatorname{kei}\left(a \eta n_{j}\right)+\operatorname{bei}\left(a \xi n_{j}\right) \operatorname{ker}\left(a \eta n_{j}\right)\right]\right. \\
\left.+\operatorname{Im}\left[\int_{0}^{\infty} s g_{j}(s / a) J_{0}(\xi s) J_{0}(\eta s) d s\right]\right\} .
\end{gathered}
$$

Returning to (22) and (23), we deduce that the original system of coupled dual integral equations (19) and (20) admit the representations

$$
\left.\begin{array}{l}
(\alpha / s) F_{1}(s)=\frac{\pi a}{2 s}\left\{\Psi_{1}(1) J_{1}(s a)-\int_{0}^{1} J_{1}(a \xi s) \frac{d}{d \xi}\left[\frac{\Psi_{1}(\xi)}{\sqrt{ }(\xi)}\right] \xi d \xi\right\} \\
(\beta / s) F_{2}(s)=\frac{\pi a}{2 s}\left\{\Psi_{2}(1) J_{1}(s a)-\int_{0}^{1} J_{1}(a \xi s) \frac{d}{d \xi}\left[\frac{\Psi_{2}(\xi)}{\sqrt{ }(\xi)}\right] \xi d \xi\right\}
\end{array}\right\}
$$

where $0<s<\infty$ and $\Psi_{j}$ are linked to $\phi_{j}$ for $j=1,2$ through (30). The remaining functions $F_{j}(s)(j=3,4)$ can be found from the algebraic equations (18). The forms of the equations (35) are particularly suited for treating the singular terms of the moments and stress resultants, since the integration in a small region surrounding the crack tip can be performed analytically. The details will be discussed in the following section.

Stress resultants and moments near crack tip. The mathematical theory of crack propagation [1] is mainly concerned with the state of affairs in the crack tip region, where extension of the crack is imminent. Within the framework of the theory of elasticity for an initially sharp crack, the stress resultants and moments at the ends of the crack exhibit mathematical singularities. It is the singular character of these quantities that has been shown to play a major role in developing the criterion of brittle fracture.

With reference to (35), it is not difficult to verify that only the leading terms identified with $\Psi_{j}(1)(j=1,2)$ contribute to the singular portion of $N_{x}, N_{y}, \ldots$, and $M_{x}, M_{y}, \ldots$ The desired explicit expressions of $F_{j}(s)(j=1,2, \ldots, 4)$ are

$$
\alpha F_{1}(s)=\frac{1}{2} \pi a \Psi_{1}(1) J_{1}(s a)+\ldots, \quad F_{2}(s)=\frac{1}{2} \pi a \Psi_{2}(1) J_{1}(s a)+\ldots
$$

and

$$
\left.\begin{array}{rl}
(1-v) F_{3}(s) & =-\frac{1}{2} \pi a\left[\left(\beta^{2}-v\right) \Psi_{1}(1)+\left(\alpha^{2}-v\right) \Psi_{2}(1)\right] J_{1}(s a)+\ldots \\
F_{4}(s) & =-\frac{1}{2} \pi a\left[\Psi_{1}(1)-\Psi_{2}(1)\right] J_{1}(s a)+\ldots
\end{array}\right\}
$$

which are derived from (18). With a view toward exhibiting the unbounded portion of the solution at the crack tips, (36) and (37) are put into (14) and (15) with $\Psi_{j}(1)$ replaced by $\phi_{j}(1)$ 
by means of (30). The results are then expanded asymptotically for large values of $s$. This gives

$$
\left.\begin{array}{rl}
N_{x}^{c} & =\frac{\pi \dot{\lambda}^{4} a}{4} R D\left[-\frac{1}{4} \lambda^{2} y^{2} \phi_{1}(1)+(1-s y) \phi_{2}(1)+O\left(\frac{1}{s}\right)\right] \exp (-s y) J_{1}(a s)+\ldots, \\
N_{y}^{c} & =\frac{\pi \dot{\lambda}^{4} a}{4} R D\left[\frac{1}{4} \lambda^{2} y^{2} \phi_{1}(1)+(1+s y) \phi_{2}(1)+O\left(\frac{1}{s}\right)\right] \exp (-s y) J_{0}(a s)+\ldots, \\
N_{x y}^{s} & =\frac{\pi \lambda^{4} a}{4} R D\left[\frac{1}{4} \lambda^{2} y^{2} \phi_{1}(1)+s y \phi_{2}(1)+O\left(\frac{1}{s}\right)\right] \exp (-s y) J_{1}(a s)+\ldots,
\end{array}\right\}
$$

and

$$
\left.\begin{array}{rl}
M_{x}^{c} & =-\frac{\pi(1-v) \lambda^{2} a}{4} D\left[(1-s y) \phi_{1}(1)+\frac{1}{4} \lambda^{2} y^{2} \phi_{2}(1)+o\left(\frac{1}{s}\right)\right] \exp (-s y) J_{1}(a s)+\ldots, \\
M_{y}^{c} & \left.=\frac{\pi \lambda^{2} a}{4} D\left\{[3+v-(1-v) s y] \phi_{1}(1)+\frac{1}{4}(1-v) \lambda^{2} y^{2} \phi_{2}(1)+o\left(\frac{1}{s}\right)\right\} \exp (-s y) J_{0}(a s)+\ldots,\right\} \\
M_{x y}^{s} & =\frac{\pi \lambda^{2} a}{4} D\left\{[2-(1-v) s y] \phi_{1}(1)+\frac{1}{4}(1-v) \lambda^{2} y^{2} \phi_{2}(1)+O\left(\frac{1}{s}\right)\right\} \exp (-s y) J_{1}(a s)+\ldots
\end{array}\right\}
$$

By recourse to (11) and with the aid of known Bessel integral-identities [9], the membrane stress resultants and moments may be evaluated in closed form in terms of elementary functions of the polar coordinates $(r, \theta),\left(r_{1}, \theta_{1}\right)$ and $\left(r_{2}, \theta_{2}\right)$, as indicated in Fig. 2. The computations

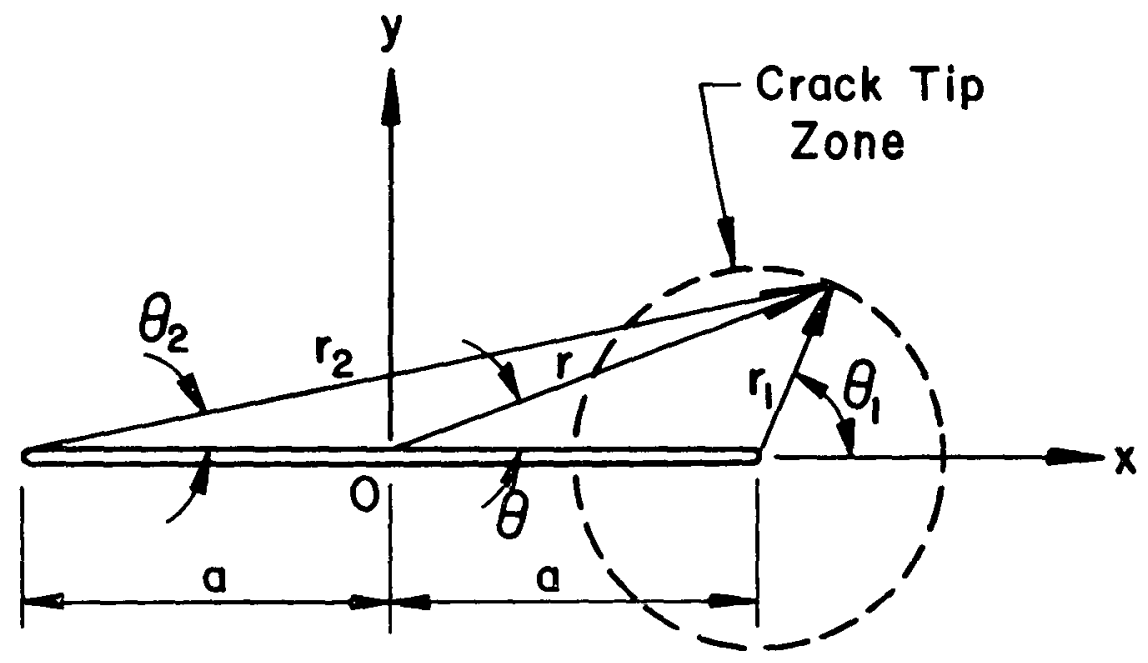

FIG. 2. Polar Coordinates measured from the Crack. 
just described lead to the expressions:

$$
\begin{aligned}
N_{x}= & -\frac{\lambda^{4} a}{2 \sqrt{ }\left(r_{1} r_{2}\right)} R D\left[\left\{\frac{1}{4} \lambda^{2} y^{2} \phi_{1}(1)-\phi_{2}(1)\right\}\left\{\frac{\sqrt{ }\left(r_{1} r_{2}\right)}{a}+\frac{r}{a} \cos \left[\theta-\frac{1}{2}\left(\theta_{1}+\theta_{2}\right)\right]\right\}\right. \\
& \left.+\phi_{2}(1)\left\{\frac{a y}{r_{1} r_{2}} \sin \left[\frac{3}{2}\left(\theta_{1}+\theta_{2}\right)\right]\right\}\right]+O(1), \\
N_{y}= & \frac{\lambda^{4} a}{2 \sqrt{ }\left(r_{1} r_{2}\right)} R D\left[\left\{\frac{1}{4} \lambda^{2} y^{2} \phi_{1}(1)+\phi_{2}(1)\right\}\left\{\frac{\sqrt{ }\left(r_{1} r_{2}\right)}{a}+\frac{r}{a} \cos \left[\theta-\frac{1}{2}\left(\theta_{1}+\theta_{2}\right)\right]\right\}\right. \\
& \left.+\phi_{2}(1)\left\{\frac{a y}{r_{1} r_{2}} \sin \left[\frac{3}{2}\left(\theta_{1}+\theta_{2}\right)\right]\right\}\right]+O(1), \\
N_{x y}= & \frac{\lambda^{4} a}{2 \sqrt{ }\left(r_{1} r_{2}\right)} R D\left[\frac{1}{4} \lambda^{2} y^{2} \phi_{1}(1)\left\{\frac{r}{a} \sin \left[\theta-\frac{1}{2}\left(\theta_{1}+\theta_{2}\right)\right]\right\}\right. \\
& \left.+\phi_{2}(1)\left\{\frac{a y}{r_{1} r_{2}} \cos \left[\frac{3}{2}\left(\theta_{1}+\theta_{2}\right)\right]\right\}\right]+O(1),
\end{aligned}
$$

and

$$
\begin{aligned}
M_{x}= & -\frac{(1-v) \lambda^{2} a}{2 \sqrt{ }\left(r_{1} r_{2}\right)} D\left[\left\{\phi_{1}(1)+\frac{1}{4} \lambda^{2} y^{2} \phi_{2}(1)\right\}\left\{\frac{\sqrt{ }\left(r_{1} r_{2}\right)}{a}+\frac{r}{a} \cos \left[\theta-\frac{1}{2}\left(\theta_{1}+\theta_{2}\right)\right]\right\}\right. \\
& \left.-\phi_{1}(1)\left\{\frac{a y}{r_{1} r_{2}} \sin \left[\frac{3}{2}\left(\theta_{1}+\theta_{2}\right)\right]\right\}\right]+O(1), \\
M_{y}= & \frac{\lambda^{2} a}{2 \sqrt{ }\left(r_{1} r_{2}\right)} D\left[\left\{(3+v) \phi_{1}(1)+\frac{1}{4}(1-v) \lambda^{2} y^{2} \phi_{2}(1)\right\}\left\{\frac{\sqrt{ }\left(r_{1} r_{2}\right)}{a}+\frac{r}{a} \cos \left[\theta-\frac{1}{2}\left(\theta_{1}+\theta_{2}\right)\right]\right\}\right. \\
& \left.-(1-v) \phi_{1}(1)\left\{\frac{a y}{r_{1} r_{2}} \sin \left[\frac{3}{2}\left(\theta_{1}+\theta_{2}\right)\right]\right\}\right]+O(1), \\
M_{x y}= & \frac{\lambda^{2} a}{2 \sqrt{ }\left(r_{1} r_{2}\right)} D\left[\left\{2 \phi_{1}(1)+\frac{1}{4}(1-v) \dot{\lambda}^{2} y^{2} \phi_{2}(1)\right\}\left\{\frac{r}{a} \sin \left[\theta-\frac{1}{2}\left(\theta_{1}+\theta_{2}\right)\right]\right\}\right. \\
& \left.-(1-v) \phi_{1}(1)\left\{\frac{a y}{r_{1} r_{2}} \cos \left[\frac{3}{2}\left(\theta_{1}+\theta_{2}\right)\right]\right\}\right]+O(1) .
\end{aligned}
$$

Note that all the quantities in (40) and (41) grow beyond bounds as $r_{1} r_{2} \rightarrow 0$. The order of the singularities at the crack tips inherent in the stress resultants is the same as that of the moments. The latter conclusion is consistent with the singular solutions obtained for the stretching and being of flat plates.

In order to expose the pertinent parameters, which are used in the current theory of brittle fracture, attention will be restricted to a small region embracing the right hand side crack vertex $(a, 0)$, as shown in Fig. 2. By taking the limits $r \rightarrow a, \theta \rightarrow 0, r_{2} \rightarrow 2 a$ and $\theta_{2} \rightarrow 0$, 
(40) and (41) further reduce to

$$
\left.\begin{array}{r}
N_{x}=\frac{k_{1}}{\sqrt{\left(2 r_{1}\right)}} \cos \frac{\theta_{1}}{2}\left[1-\sin \frac{\theta_{1}}{2} \sin \frac{3 \theta_{1}}{2}\right], \\
N_{y}=\frac{k_{1}}{\sqrt{\left(2 r_{1}\right)}} \cos \frac{\theta_{1}}{2}\left[1+\sin \frac{\theta_{1}}{2} \sin \frac{3 \theta_{1}}{2}\right], \\
N_{x y}=\frac{k_{1}}{\sqrt{\left(2 r_{1}\right)}} \cos \frac{\theta_{1}}{2} \sin \frac{\theta_{1}}{2} \cos \frac{3 \theta_{1}}{2},
\end{array}\right\}
$$

and

$$
\left.\begin{array}{c}
M_{x}=-\frac{K_{1}}{\sqrt{\left(2 r_{1}\right)}}(1-v) \cos \frac{\theta_{1}}{2}\left[1-\sin \frac{\theta_{1}}{2} \sin \frac{3 \theta_{1}}{2}\right], \\
M_{y}=-\frac{K_{1}}{\sqrt{\left(2 r_{1}\right)}} \cos \frac{\theta_{1}}{2}\left[(3+v)-(1-v) \sin \frac{\theta_{1}}{2} \sin \frac{3 \theta_{1}}{2}\right], \\
M_{x y}=-\frac{K_{1}}{\sqrt{\left(2 r_{1}\right)}} \sin \frac{\theta_{1}}{2}\left[2+(1-v) \cos \frac{\theta_{1}}{2} \cos \frac{3 \theta_{1}}{2}\right] .
\end{array}\right\}
$$

Here, the coefficients $k_{1}$ and $K_{1}$ may be referred to as the intensity-factors of their respective stress resultant and moment fields. For the present problem of a spherical shell, $k_{1}$ and $K_{1}$ are coupled through the functions $\phi_{j}(1)(j=1,2)$ appearing in (30) as follows:

$$
k_{1}=\frac{1}{2} \lambda^{4} R D \phi_{2}(1) \sqrt{ } a, \quad K_{1}=\frac{1}{2} \lambda^{2} D \phi_{1}(1) \sqrt{ } a .
$$

In other words, an interaction exists between bending and stretching such that application of one type of loading induces stresses of the other type.

The foregoing results illustrate the qualitative features of the spherical shell solution. It is seen from (42) and (43) that the $r_{1}$ - and $\theta_{1}$-dependence coincide with those found for the stretching and bending of flat plates. Hence, the curvature effect governed by $\lambda$ enters into the local solution only through the intensity-factors $k_{1}$ and $K_{1}$. It is now apparent that as the curvature of the shell becomes increasingly large, the formal appearance of (42) and (43) remains unchanged, while $k_{1}$ and $K_{1}$ are de-coupled to the separate solutions for an initially flat plate under stretching and bending. Another important point to be noted is that the bending part of the solution is based upon the satisfaction of the approximate Kirchhoff shear condition on the crack edge. Thus, the differences between the approximate and exact boundary conditions on the crack might be anticipated to be very similar to the changes found between [11] and [5, 12] for the bending of an initially flat plate. $\dagger$ In both types of solution, i.e., $[11]$ and $[5,12]$, the stress singularity remained of the inverse square-root type but in the case where the physical boundary conditions are actually satisfied on the crack $[5,12]$, the distribution of bending stress around the crack point became identical with that predicted for an initially flat plate subjected solely to extension. The similar improved solution for the

$\dagger$ The plate bending theory used in $[11]$ was originated by Poisson-Kirchhoff and in $[5,12]$ by Reissner. 
spherical shell, however, is a considerably more difficult problem. Nevertheless, it is reasonable to assume that the difference reflected for the flat plate case will also hold if a more refined theory were developed for the spherical shell.

The numerical results of $k_{1}$ and $K_{1}$ are obtained by solving the coupled Fredholm integral equations (31) on a computer. Two examples of basic interest are treated:

Case (1). Stretching load: $N(x)=N_{0}, M(x)=0$,

$$
k_{1}=\Phi_{2}(1) N_{0} \sqrt{ } a, \quad K_{1}=\frac{h}{\sqrt{\left[12\left(1-v^{2}\right)\right]}} \Phi_{1}(1) N_{0} \sqrt{ } a,
$$

where

$$
\Phi_{j}(1)=\left(\alpha_{2} / n_{0}\right) \phi_{j}(1) \quad(j=1,2) .
$$

Case (2). Bending load: $N(x)=0, M(x)=M_{0}$,

$$
k_{1}=\frac{\sqrt{ }\left[12\left(1-v^{2}\right)\right]}{h(3+v)} \Phi_{2}(1) M_{0} \sqrt{ } a, \quad K_{1}=\frac{1}{3+v} \Phi_{1}(1) M_{0} \sqrt{ } a
$$

where

$$
\Phi_{j}(1)=-\left(\alpha_{1} / m_{0}\right) \phi_{j}(1) \quad(j=1,2) .
$$

Graphs of the normalized intensity-factors versus the curvature parameter $\lambda a$ are plotted in Figs. 3 and 4. The solid curves represent the solutions of the coupled Fredholm integral equations (31), while the dotted curves correspond to the results given in [4].

In Case (1), the crack is opened out by a uniform stretching load $N_{0}$. Because of the interaction between extension and bending, both $k_{1}$ and $K_{1}$ exist as in (45). The solid curves in Figs. 3a and 3b show that the magnitude of $k_{1}$ and $K_{1}$ increases monotonically with $\lambda a$. Further, the values of the membrane stress-intensity factor $k_{1}$ are much higher than the bending moment-intensity factor $K_{1}$. In Fig. 3a, the dotted curve taken from [4] is accurate only for $0 \leqq \lambda a \leqq 0.46$. Beyond this range, it differs significantly from the more refined results indicated by the solid curve. For the bending moment-intensity factor $K_{1}$ in Fig. 3b, the dotted curve is observed to be valid only for values of $\lambda a$ up to 0.26 . As $\lambda a$ increases, the solution in [4] suggests that there is a change in the mode of bending behaviour of the shell since $K_{1}$ changes sign.

Similar results for the case when the crack is subjected to a uniform bending moment $M_{0}$ are displayed in Figs. $4 \mathrm{a}$ and $4 \mathrm{~b}$. In this example, the moment-intensity factor $K_{1}$ dominates whereas the strength of the membrane stress-intensity factor $k_{1}$ is relatively low. The approximate solutions of $k_{1}$ and $K_{1}$ in [4] given by the dotted curves are seen to deviate appreciably from the present results even for small values of $\lambda a$.

In general, for either type of loading, $k_{1}$ and $K_{1}$ in a spherical shell are increased over their values in a flat plate, $\lambda=0$. 


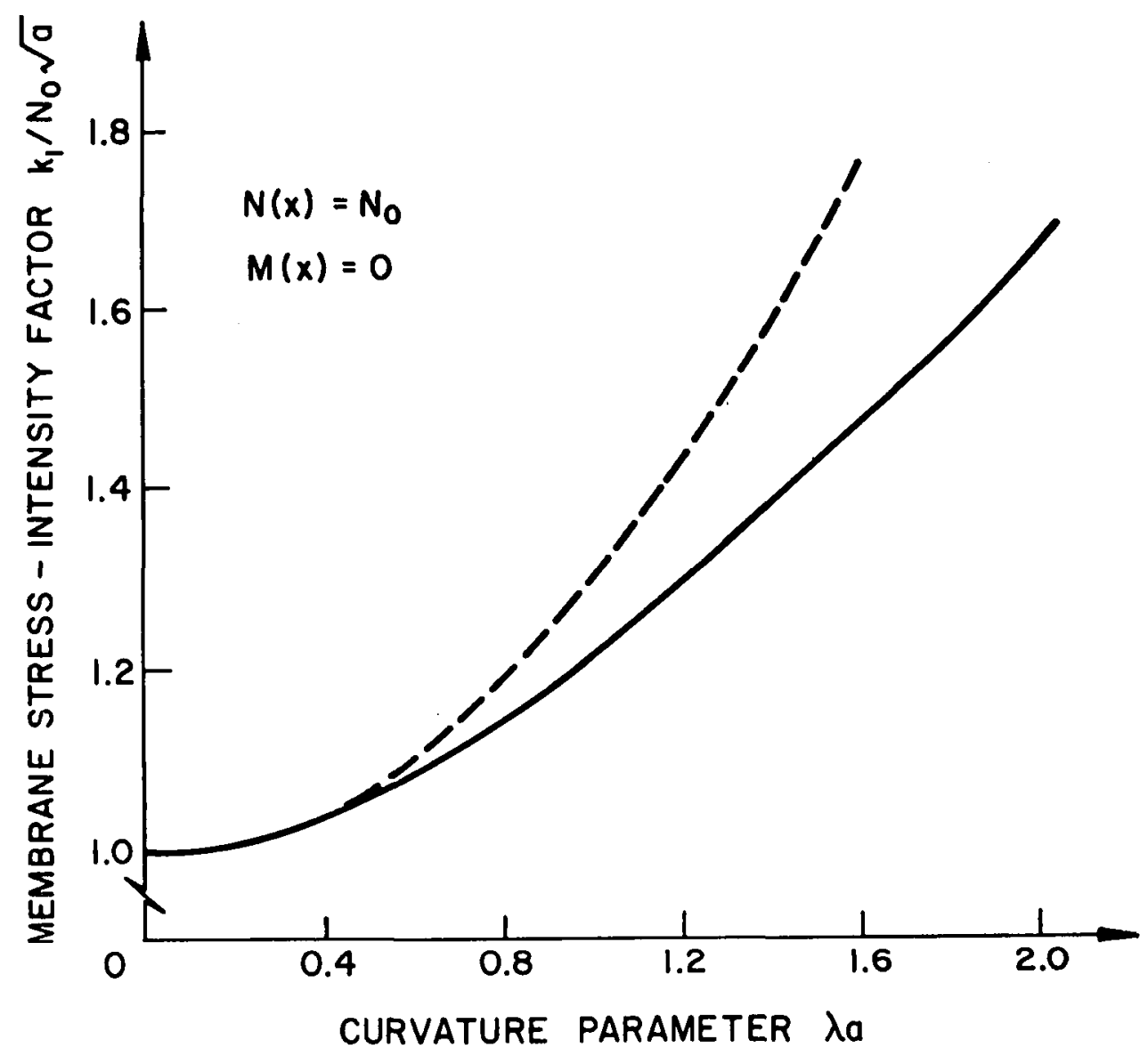

FIG. 3a. Membrane Stress-Intensity Factor Curves for Stretching Load. 


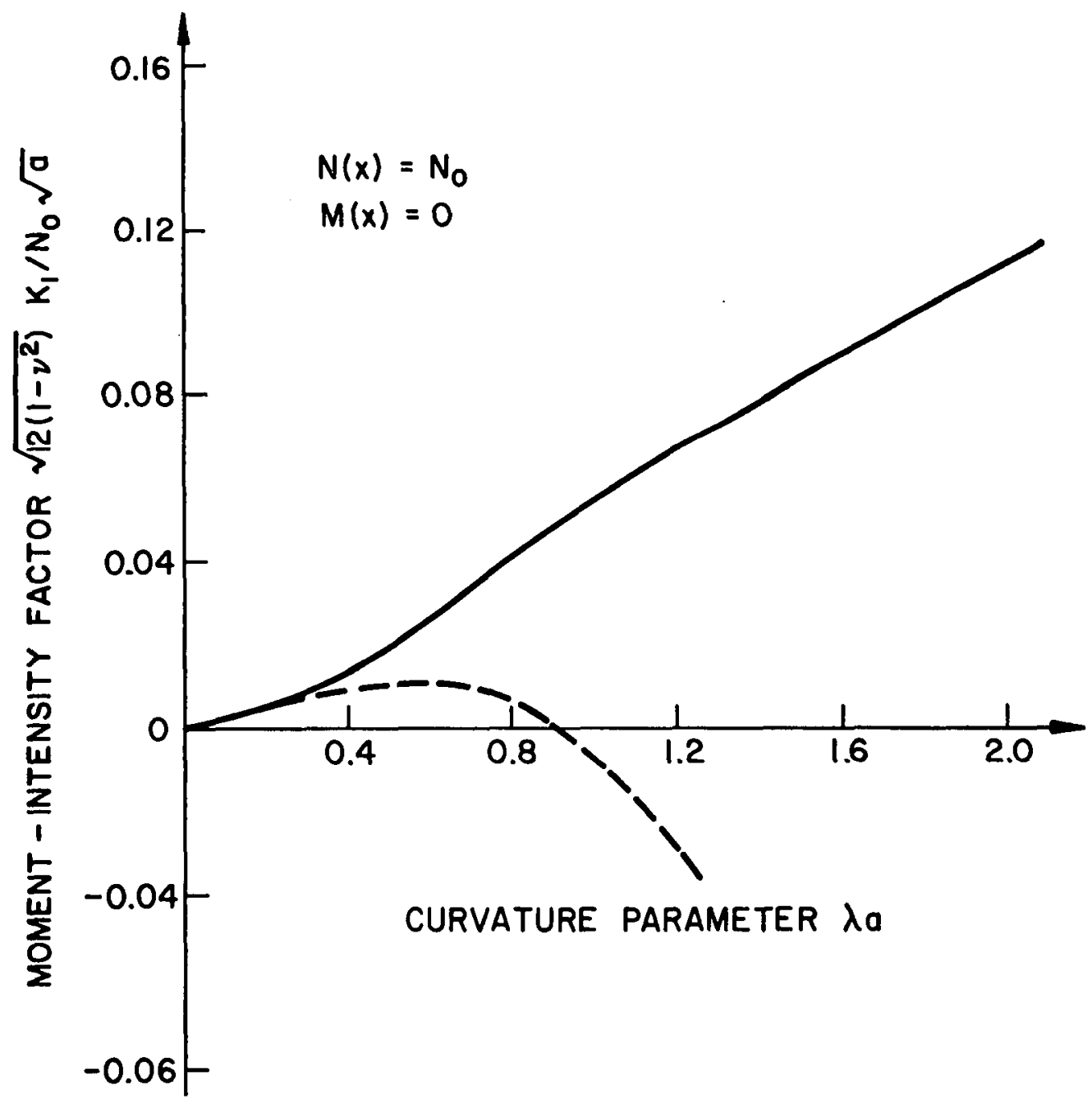

Fig. 3b. Moment-Intensity Factor Curves for Stretching Load. 


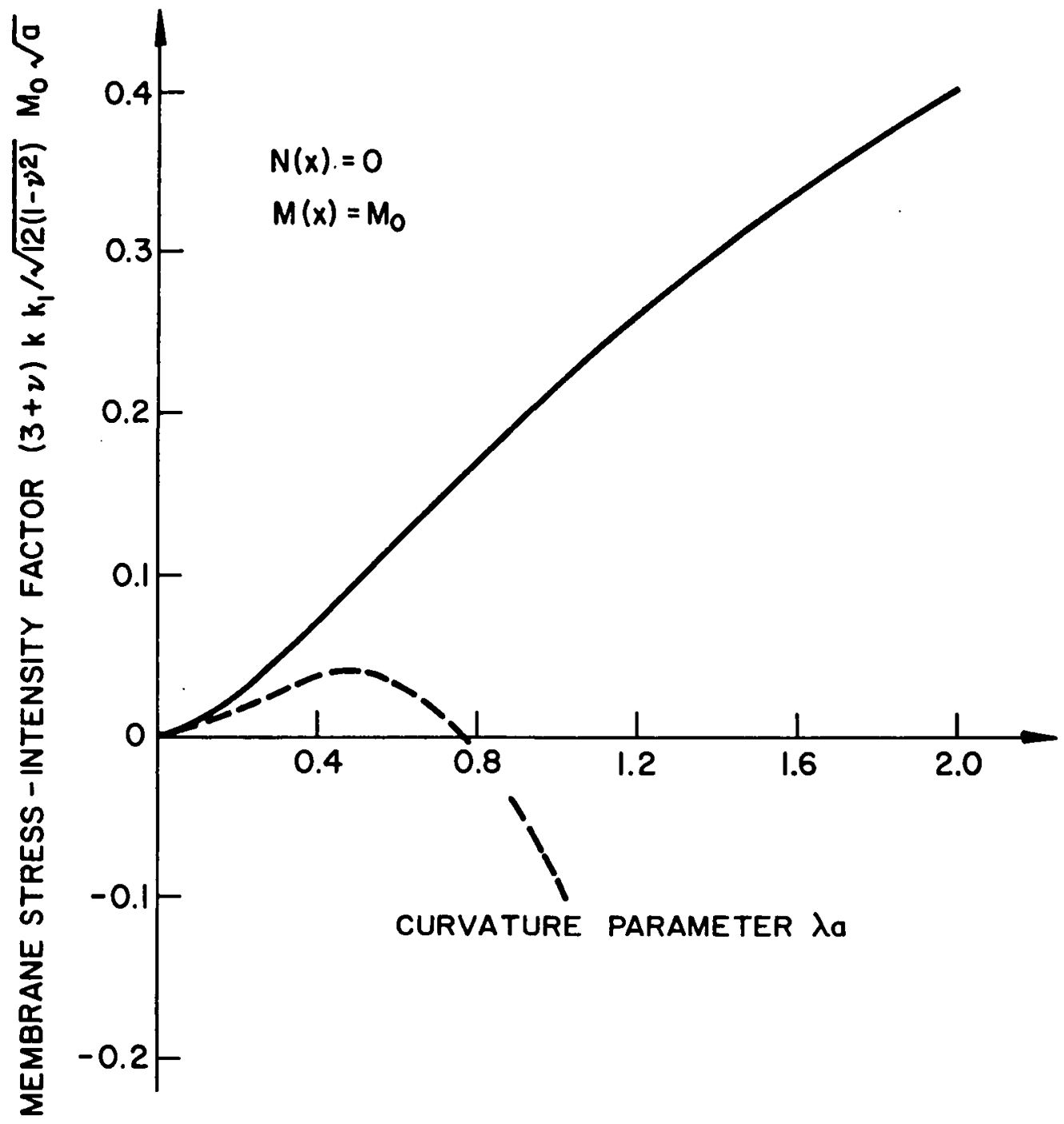

Fig. 4a. Membrane Stress-Intensity Factor Curves for Bending Load. 


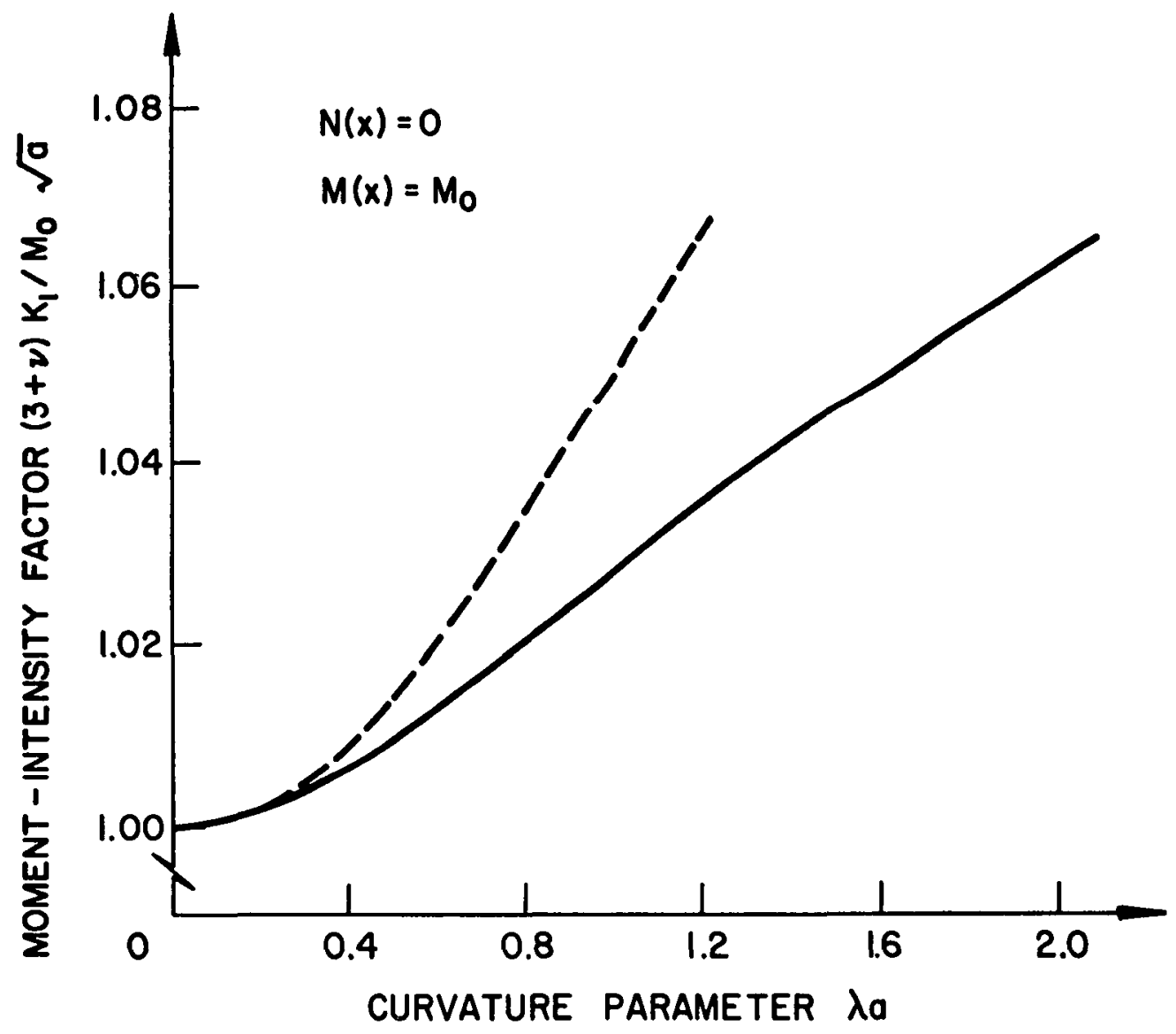

FIg. 4b. Moment-Intensity Factor Curves for Bending Load. 
Displacements and deflection slopes of crack surface. With the aim of gaining a quantitative insight into the deformed shape of the crack, it is essential to compute numerically the normal displacement $v(x, 0)$ and the deflection slope $\partial w / \partial y$ as $y \rightarrow 0$ for $-a<x<a$. More precisely, for the two examples considered earlier, numerical results have been obtained as follows:

Case (1). $N(x)=N_{0}, M(x)=0$,

$$
\begin{array}{ll}
-v(x, 0)=\frac{(\lambda+R D) n_{0}}{E h \alpha_{2}} \int_{x / a}^{1}\left(\xi^{2}-\frac{x^{2}}{a^{2}}\right)^{-\frac{1}{2}} \sqrt{ }(\xi) \Phi_{2}(\xi) d \xi & (x<a), \\
\left(\frac{\partial w}{\partial y}\right)_{y=0}=\frac{\dot{\lambda}^{2} a n_{0}}{(1-v) \alpha_{2}} \int_{x / a}^{1}\left(\xi^{2}-\frac{x^{2}}{a^{2}}\right)^{-\frac{1}{2}} \sqrt{ }(\xi) \Phi_{1}(\xi) d \xi & (x<a) .
\end{array}
$$

Case (2). $\quad N(x)=0, M(x)=M_{0}$,

$$
\begin{aligned}
& v(x, 0)=\frac{\lambda^{4} R D m_{0}}{E h \alpha_{1}} \int_{x / a}^{1}\left(\xi^{2}-\frac{x^{2}}{a^{2}}\right)^{-\frac{1}{2}} \sqrt{ }(\xi) \Phi_{2}(\xi) d \xi \quad(x<a), \\
& -\left(\frac{\partial w}{\partial y}\right)_{y=0}=\frac{\lambda^{2} a m_{0}}{(1-v) \alpha_{1}} \int_{x / a}^{1}\left(\xi^{2}-\frac{x^{2}}{a^{2}}\right)^{-\frac{1}{2}} \sqrt{ }(\xi) \Phi_{1}(\xi) d \xi \quad(x<a) .
\end{aligned}
$$

Consider the case of the crack being stretched open by a uniform load $N_{0}$. Fig. 5 shows a plot of the dimensionless form of (47) versus the normalized distance $x / a$ for various values of $\lambda a$. As $\lambda a$ increases, the normal displacement curves may be seen to increase in magnitude and to display more marked variation along the crack. Since the load is symmetric, the normal displacement is maximum at the center $x=0$ and tapers off to zero at both ends of the crack $x= \pm a$. On account of the shell curvature, the stretching load also produces rotation of the crack surface about the $x$-axis. The magnitude of this rotation decreases with the curvature parameter $\lambda a$ as indicated in Fig. $5 \mathrm{~b}$ and is zero along the entire crack for $\lambda=0$, which corresponds to the limiting case of a flat plate.

Now, let the crack be bent open by a uniform moment $M_{0}$. In this case, a considerable amount of rotation of the crack surface can be observed from Fig. 6a. The curvature parameter $\lambda a$ appears to have a little effect on the variation of $\partial w / \partial y$ for $-a<x<a$. The curves in Fig. $6 \mathrm{~b}$ represent the normal displacements of the crack caused by the bending moment $M_{0}$ and they diminish in magnitude as $\lambda \rightarrow 0$.

Conclusion. On the basis of the first-order shallow shell theory, the problem of a spherical shell weakened by a through crack subjected to stretching and bending loads is solved. The method of analysis, in which the mixed boundary value problem is reduced to the solution of two coupled Fredholm integral equations, has proved successful, by carrying out the numerical calculations on a computer. It has been found that the stresses in a shell are in general larger than those obtained in a flat plate. 


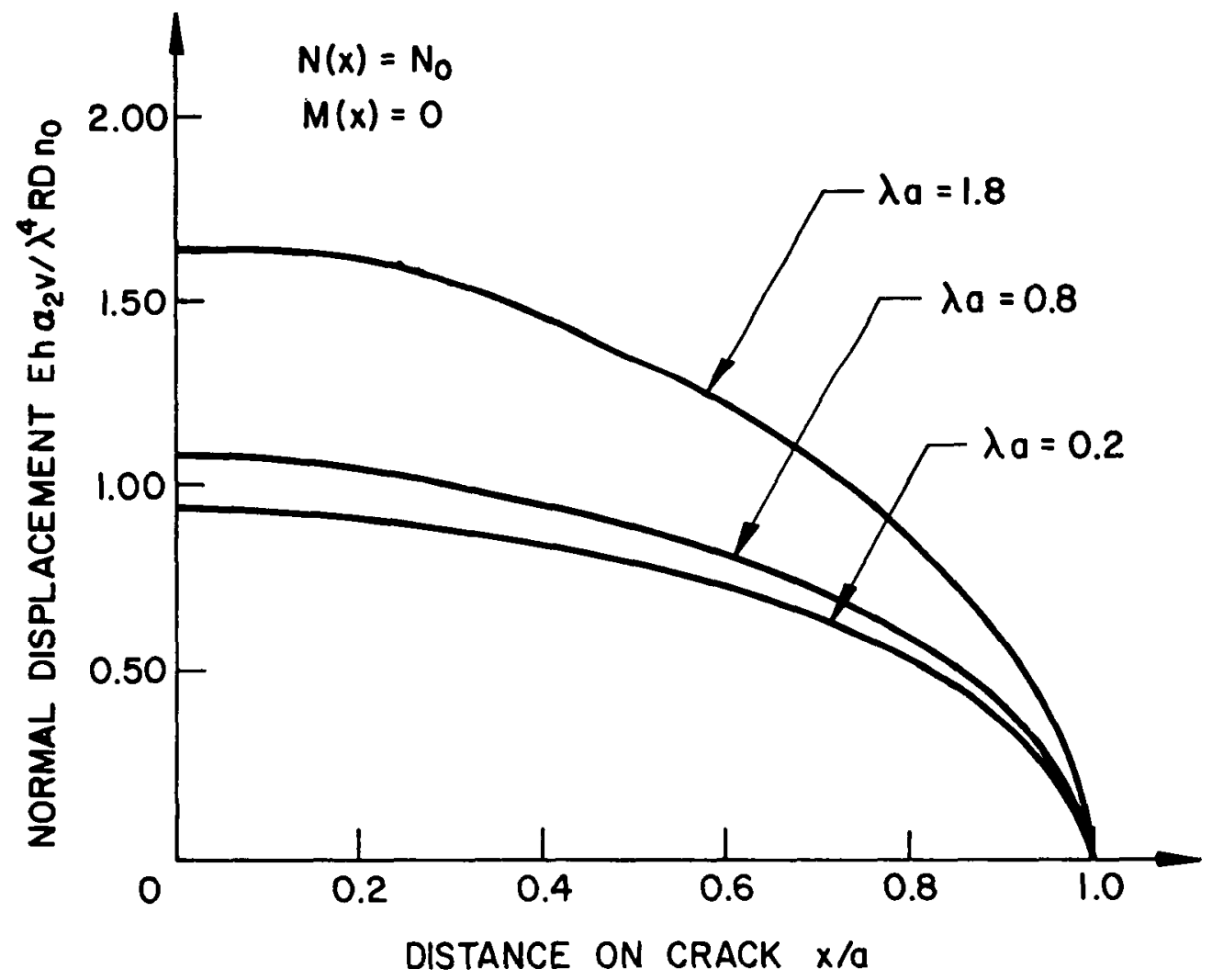

FIG. 5a. Normalized Displacement versus Distance on Crack for Stretching Load. 


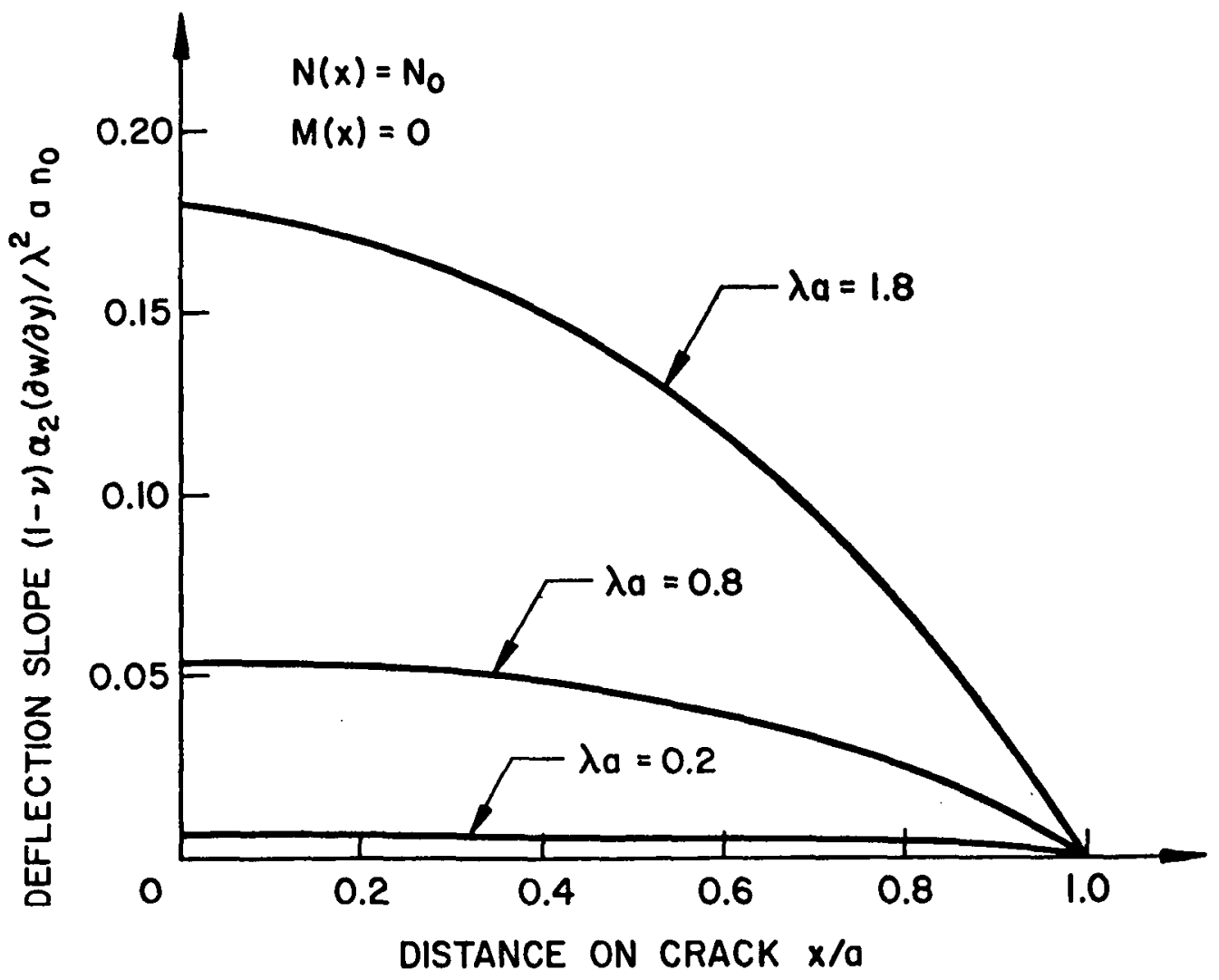

Fig. 5b. Deflection Slope as a Function of Distance on Crack for Stretching Load. 


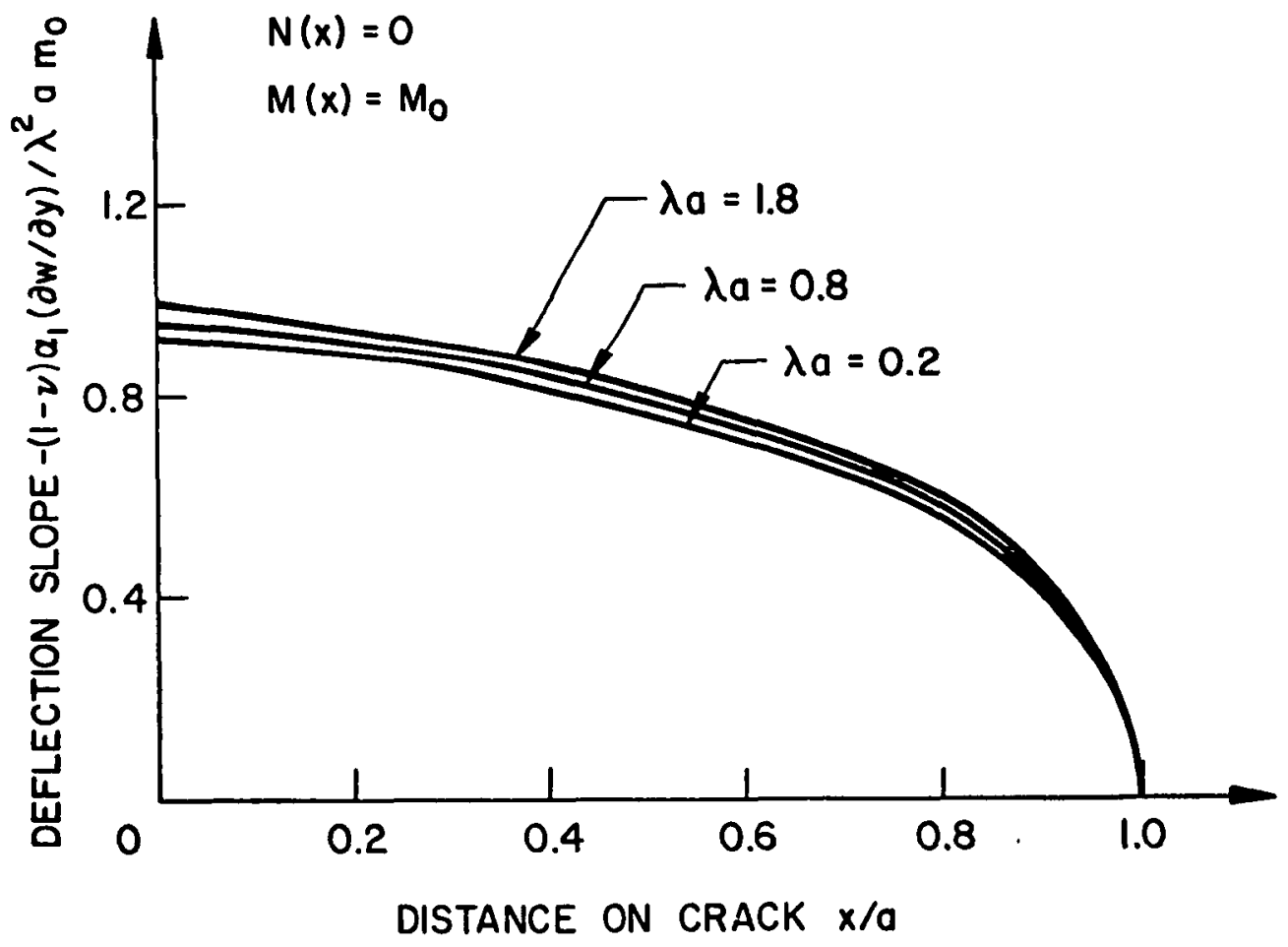

FIG. 6a. Deflection Slope as a Function of Distance on Crack for Bending Load. 


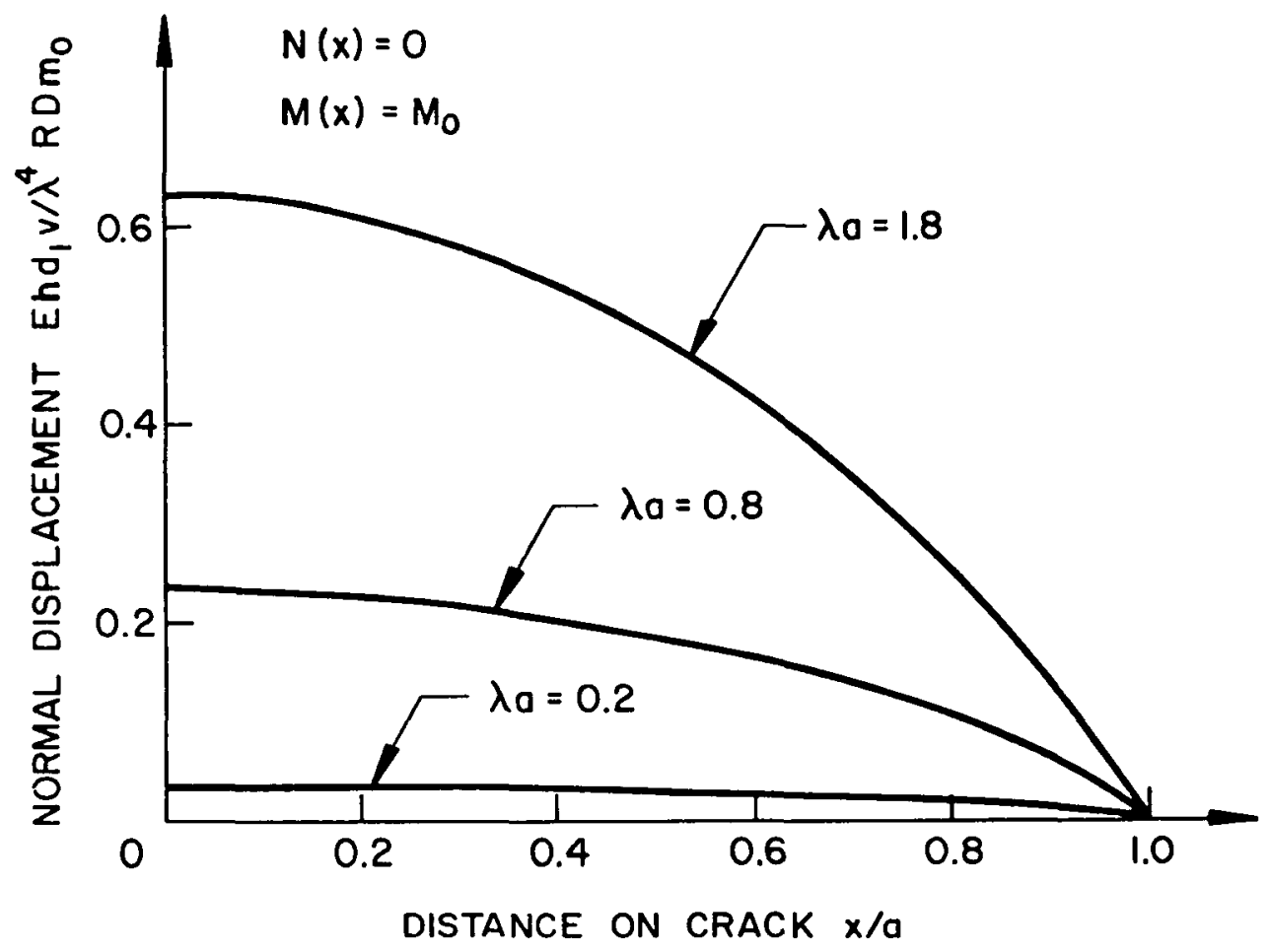

FIG. 6b. Normalized Displacement versus Distance on Crack for Bending Load. 
It should be remarked that a number of related spherical shell problems of practical interest can be solved using the same approach. Moreover, the same type of analysis can also be applied to the case of a pressurized cylindrical shell with a crack.

\section{REFERENCES}

1. G. C. Sih and H. Liebowitz, Mathematical theories of brittle fracture, Mathematical fundamentals of fracture, Vol. 2, Academic Press (New York, 1968), 67-190.

2. D. D. Ang, E. S. Folias and M. L. Williams, The bending stress in a cracked plate on an elastic foundation, J. Appl. Mech. 30 (1963), 245-251.

3. G. C. Sih and D. E. Setzer, Discussion of " The bending stress in a cracked plate on an elastic foundation ", J. Appl. Mech. 31 (1964), 365-367.

4. E. S. Folias, The stresses in a cracked spherical shell, J. Math. and Phys. 44 (1965), 164-176.

5. J. K. Knowles and N. M. Wang, On the bending of an elastic plate containing a crack, J. Math. and Phys. 39 (1960), 223-236.

6. E. Reissner, On some problems in shell theory, Structural mechanics, Proceedings of the First Symposium on Naval Structural Mechanics (1958), 74-113.

7. I. N. Sneddon, Fourier transforms, McGraw-Hill (New York, 1951), 7I-82.

8. G. C. Sih, Flexural problems of cracks in mixed media, Proceedings of the First International Conference on Fracture, 1 (1965), 391-409.

9. G. N. Watson, Theory of Bessel functions, Cambridge University Press (London, 1958). 1953).

10. R. Courant and D. Hilbert, Methods of mathematical physics, Vol. 1, Interscience (New York

11. M. L. Williams, The bending stress distribution at the base of a stationary crack, J. Appl. Mech. 28 (1961), 78-82.

12. R. J. Hartranft and G. C. Sih, Effect of plate thickness on the bending stress distribution around through cracks, J. Math. and Phys. 47 (1968), 276-291.

\section{LEHIGH UNIVERSITY}

BETHLEHEM

PenNSYlvania 